Bull. Korean Math. Soc. 47 (2010), No. 3, pp. 653-661

DOI 10.4134/BKMS.2010.47.3.653

\title{
UNIT-REGULARITY AND STABLE RANGE ONE
}

\author{
HuAnyin Chen
}

\begin{abstract}
Let $R$ be a ring, and let $\Psi(R)$ be the ideal generated by the set $\{x \in R \mid 1+s x t \in R$ is unit-regular for all $s, t \in R\}$. We show that $\Psi(R)$ has "radical-like" property. It is proven that $\Psi(R)$ has stable range one. Thus, diagonal reduction of matrices over such ideal is reduced.
\end{abstract}

\section{Introduction}

Let $R$ be an associative ring with an identity. An element $a \in R$ is regular in case there exists $x \in R$ such that $a=a x a$. If such $x$ can be chosen a unit, $a \in R$ is said to be unit-regular. A ring $R$ is unit-regular in case every element in $R$ is unit-regular. It is worth noting that a regular $\operatorname{ring} R$ is unit-regular if and only if for all finitely generated projective right $R$-modules $A, B$ and $C$, $A \oplus B \cong A \oplus C$ implies that $B \cong C$. Many authors studied unit-regular rings, e.g. $[5,7]$. As is well known, there exists a largest regular ideal $M(R)$ of a ring $R$. A natural problem is how to construct a kind of ideal to deal with unit-regularity. The motivation of this article is to extend the known results on unit-regular rings to regular ideals. We always use $\operatorname{ur}(R)$ to denote the set of all unit-regular elements in $R$. Let $\Psi(R)$ be the ideal generated by the set $\{x \in R \mid 1+s x t \in u r(R)$ for all $s, t \in R\}$. We observe that $\Psi(R)$ has "radicallike" property. That is, $M_{n}(\Psi(R))=\Psi\left(M_{n}(R)\right)$. An ideal $I$ of a ring $R$ has stable range one provided that $a R+b R=R$ with $a \in 1+I, b \in R$ implies that $a+b y \in R$ is invertible. For general theory of stable range conditions, we refer the reader to [6]. Further, we prove that $\Psi(R)$ has stable range one. As an application, the diagonal reduction of matrices over such ideal is studied.

Throughout, all rings are associative with identity. $U(R)$ and $\mathrm{GL}_{n}(R)$ denote the set of all units of $R$ and the $n$-dimensional general linear group over $R$, respectively. The symbol $\mathbb{N}$ stands for the set of all natural number.

\section{Unit-regularity}

It is well known that for any $x, y \in R, 1+x y \in U(R)$ if and only if $1+y x \in$ $U(R)$. We extend this simple fact to unit-regularity.

Received January 21, 2009; Revised April 3, 2009.

2000 Mathematics Subject Classification. 16E50, 16 U99.

Key words and phrases. unit-regularity, stable range one, diagonal reduction. 
Lemma 2.1. Let $x, y \in R$. Then $1+x y$ is unit-regular if and only if so is $1+y x$.

Proof. Suppose that $1+x y$ is unit-regular. Clearly, one checks that

$\left(\begin{array}{cc}1 & 0 \\ 0 & 1+y x\end{array}\right)=\left(\begin{array}{ll}1 & 0 \\ y & 1\end{array}\right)\left(\begin{array}{ll}1 & x \\ 0 & 1\end{array}\right)\left(\begin{array}{cc}1+x y & 0 \\ 0 & 1\end{array}\right)\left(\begin{array}{cc}1 & 0 \\ -y & 1\end{array}\right)\left(\begin{array}{cc}1 & -x \\ 0 & 1\end{array}\right)$.

Hence $\left(\begin{array}{cc}1 & 0 \\ 0 & 1+y x\end{array}\right) \in M_{2}(R)$ is unit-regular. Write

$$
\left(\begin{array}{cc}
1 & 0 \\
0 & 1+y x
\end{array}\right)=\left(\begin{array}{cc}
1 & 0 \\
0 & 1+y x
\end{array}\right)\left(\begin{array}{cc}
c_{11} & c_{12} \\
c_{21} & c_{22}
\end{array}\right)\left(\begin{array}{cc}
1 & 0 \\
0 & 1+y x
\end{array}\right)
$$

where $\left(\begin{array}{ll}c_{11} & c_{12} \\ c_{21} & c_{22}\end{array}\right) \in \mathrm{GL}_{2}(R)$. Then we get $1+y x=(1+y x) c_{22}(1+y x)$. Let $E=\left(\begin{array}{cc}1 & 0 \\ 0 & 1+y x\end{array}\right)\left(\begin{array}{ll}c_{11} & c_{12} \\ c_{21} & c_{22}\end{array}\right)$. Since

$$
\left(\begin{array}{cc}
1 & 0 \\
0 & 1+y x
\end{array}\right)\left(\begin{array}{cc}
1 & 0 \\
0 & c_{22}
\end{array}\right)+\left(\begin{array}{cc}
0 & 0 \\
0 & 1-(1+y x) c_{22}
\end{array}\right)=\left(\begin{array}{ll}
1 & 0 \\
0 & 1
\end{array}\right),
$$

we get

$E\left(\begin{array}{ll}c_{11} & c_{12} \\ c_{21} & c_{22}\end{array}\right)^{-1}\left(\begin{array}{cc}1 & 0 \\ 0 & c_{22}\end{array}\right)\left(I_{2}-E\right)+\left(\begin{array}{cc}0 & 0 \\ 0 & 1-(1+y x) c_{22}\end{array}\right)\left(I_{2}-E\right)=I_{2}-E$.

This implies that

$$
\begin{aligned}
& E+\left(\begin{array}{cc}
0 & 0 \\
0 & 1-(1+y x) c_{22}
\end{array}\right)\left(I_{2}-E\right) \\
= & I_{2}-E\left(\begin{array}{cc}
c_{11} & c_{12} \\
c_{21} & c_{22}
\end{array}\right)^{-1}\left(\begin{array}{cc}
1 & 0 \\
0 & c_{22}
\end{array}\right)\left(I_{2}-E\right) \in \mathrm{GL}_{2}(R) .
\end{aligned}
$$

Set $\left(\begin{array}{ll}z_{11} & z_{12} \\ z_{21} & z_{22}\end{array}\right)=\left(I_{2}-E\right)\left(\begin{array}{lll}c_{11} & c_{12} \\ c_{21} & c_{22}\end{array}\right)^{-1}$. Then

$$
\left(\begin{array}{cc}
1 & 0 \\
0 & 1+y x
\end{array}\right)+\left(\begin{array}{cc}
0 & 0 \\
0 & 1-(1+y x) c_{22}
\end{array}\right)\left(\begin{array}{cc}
z_{11} & z_{12} \\
z_{21} & z_{22}
\end{array}\right) \in \mathrm{GL}_{2}(R)
$$

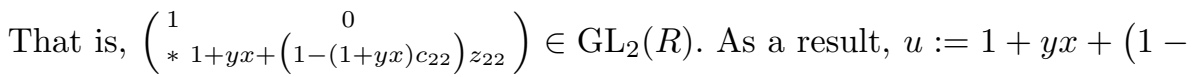
$\left.(1+y x) c_{22}\right) z_{22} \in U(R)$. Hence, $1+y x=e u$, where $e=(1+y x) c_{22} \in R$ is an idempotent. Therefore $1+y x \in R$ is unit-regular. The converse is symmetric.

Lemma 2.2. An element $a \in R$ is unit-regular if and only if $\operatorname{diag}(a, 1, \ldots, 1)$ $\in M_{n}(R)$ is unit-regular.

Proof. It is an immediate consequence of [7, Theorem 4].

Let $\Phi(R)=\{x \in R \mid 1+s x t \in R$ is unit-regular for all $s, t \in R\}$, and let $\Psi(R)$ be the ideal generated by the set $\Phi(R)$. We list some examples of such ideal. $\Psi(\mathbb{Z})=0 ; \Psi(\mathbb{Z} \oplus \mathbb{Z} / 2 \mathbb{Z})=0 \oplus \mathbb{Z} / 2 \mathbb{Z}$, and that

$$
\Psi\left(\left(\begin{array}{cc}
\mathbb{Z} / 2 \mathbb{Z} & \mathbb{Z} / 2 \mathbb{Z} \\
0 & \mathbb{Z} / 2 \mathbb{Z}
\end{array}\right)\right)=\left(\begin{array}{cc}
\mathbb{Z} / 2 \mathbb{Z} & \mathbb{Z} / 2 \mathbb{Z} \\
0 & \mathbb{Z} / 2 \mathbb{Z}
\end{array}\right)
$$

Theorem 2.3. Let $R$ be a ring. Then $\Psi\left(M_{n}(R)\right)=M_{n}(\Psi(R))$ for all $n \in \mathbb{N}$. 
Proof. Given any $A \in \Psi\left(M_{n}(R)\right)$, we have $A=A_{1}+\cdots+A_{m}(m \in \mathbb{N})$ with each $A_{i} \in \Phi\left(M_{n}(R)\right)$. Write $A_{1}=\left(a_{i j}\right)$. For any $r \in R$, we have

$$
I_{n}+A_{1}\left(\begin{array}{cccc}
r & 0 & \cdots & 0 \\
0 & 0 & \cdots & 0 \\
\vdots & \vdots & \ddots & \vdots \\
0 & 0 & \cdots & 0
\end{array}\right)=\left(\begin{array}{cccc}
1+a_{11} r & 0 & \cdots & 0 \\
a_{21} r & 1 & \cdots & 0 \\
\vdots & \vdots & \ddots & \vdots \\
a_{n 1} r & 0 & \cdots & 1
\end{array}\right)
$$

is unit-regular. Clearly, there exists $V \in \mathrm{GL}_{n}(R)$ such that

$$
\left(\begin{array}{cccc}
1+a_{11} r & 0 & \cdots & 0 \\
a_{21} r & 1 & \cdots & 0 \\
\vdots & \vdots & \ddots & \vdots \\
a_{n 1} r & 0 & \cdots & 1
\end{array}\right)=\left(\begin{array}{cccc}
1+a_{11} r & 0 & \cdots & 0 \\
0 & 1 & \cdots & 0 \\
\vdots & \vdots & \ddots & \vdots \\
0 & 0 & \cdots & 1
\end{array}\right) V
$$

Hence $\left(\begin{array}{cccc}1+a_{11} r & 0 & \cdots & 0 \\ 0 & 1 & \cdots & 0 \\ \vdots & \vdots & \ddots & \vdots \\ 0 & 0 & \cdots & 1\end{array}\right) \in M_{n}(R)$ is unit-regular, and then so is $1+a_{11} r \in R$ from Lemma 2.2. It follows by Lemma 2.1 that $a_{11} \in \Phi(R)$. Likewise, we prove that each $a_{i j} \in \Phi(R)$. Hence, $A_{1} \in M_{n}(\Psi(R))$. Similarly, $A_{2}, \ldots, A_{m} \in$ $M_{n}(\Psi(R))$. Consequently, $A=A_{1}+\cdots+A_{m} \in M_{n}(\Psi(R))$. We infer that $\Psi\left(M_{n}(R)\right) \subseteq M_{n}(\Psi(R))$.

Given any $\left(a_{i j}\right) \in M_{n}(\Psi(R))$, then each $a_{i j} \in \Psi(R)$. Write $a_{i j}=b_{1}+\cdots+b_{k}$ with each $b_{s} \in \Phi(R)(1 \leq s \leq k)$. For any $\left(r_{i j}\right) \in M_{n}(R)$, we have

$$
I_{n}+\left(\begin{array}{cccc}
b_{1} & 0 & \cdots & 0 \\
0 & 0 & \cdots & 0 \\
\vdots & \vdots & \ddots & \vdots \\
0 & 0 & \cdots & 0
\end{array}\right)\left(r_{i j}\right)=\left(\begin{array}{cccc}
1+b_{1} r_{11} & b_{1} r_{12} & \cdots & b_{1} r_{1 n} \\
0 & 1 & \cdots & 0 \\
\vdots & \vdots & \ddots & \vdots \\
0 & 0 & \cdots & 1
\end{array}\right)
$$

Clearly, there is $U \in \mathrm{GL}_{n}(R)$ such that

$$
U\left(I_{n}+\left(\begin{array}{cccc}
b_{1} & 0 & \cdots & 0 \\
0 & 0 & \cdots & 0 \\
\vdots & \vdots & \ddots & \vdots \\
0 & 0 & \cdots & 0
\end{array}\right)\left(r_{i j}\right)\right)=\left(\begin{array}{cccc}
1+b_{1} r_{11} & 0 & \cdots & 0 \\
0 & 1 & \cdots & 0 \\
\vdots & \vdots & \ddots & \vdots \\
0 & 0 & \cdots & 1
\end{array}\right) .
$$

As $1+b_{1} r_{11} \in R$ is unit-regular, by Lemma 2.2 , we have

$$
U\left(I_{n}+\left(\begin{array}{cccc}
b_{1} & 0 & \cdots & 0 \\
0 & 0 & \cdots & 0 \\
\vdots & \vdots & \ddots & \vdots \\
0 & 0 & \cdots & 0
\end{array}\right)\left(r_{i j}\right)\right) \in M_{n}(R)
$$


is unit-regular, and thus so is

$$
I_{n}+\left(\begin{array}{cccc}
b_{1} & 0 & \cdots & 0 \\
0 & 0 & \cdots & 0 \\
\vdots & \vdots & \ddots & \vdots \\
0 & 0 & \cdots & 0
\end{array}\right)\left(r_{i j}\right)
$$

Thus, $\left(\begin{array}{cccc}b_{1} & 0 & \cdots & 0 \\ 0 & 0 & \cdots & 0 \\ \vdots & \vdots & \ddots & \vdots \\ 0 & 0 & \cdots & 0\end{array}\right) \in \Psi\left(M_{n}(R)\right)$. Likewise, $\left(\begin{array}{cccc}b_{i} & 0 & \cdots & 0 \\ 0 & 0 & \cdots & 0 \\ \vdots & \vdots & \ddots & \vdots \\ 0 & 0 & \cdots & 0\end{array}\right) \in \Psi\left(M_{n}(R)\right)$ for all

$i$. We infer that $\left(\begin{array}{cccc}a_{11} & 0 & \cdots & 0 \\ 0 & 0 & \cdots & 0 \\ \vdots & \vdots & \ddots & \vdots \\ 0 & 0 & \cdots & 0\end{array}\right) \in \Psi\left(M_{n}(R)\right)$. Analogously,

$$
\left(\begin{array}{cccc}
0 & a_{12} & \cdots & 0 \\
0 & 0 & \cdots & 0 \\
\vdots & \vdots & \ddots & \vdots \\
0 & 0 & \cdots & 0
\end{array}\right), \ldots,\left(\begin{array}{cccc}
0 & 0 & \cdots & 0 \\
0 & 0 & \cdots & 0 \\
\vdots & \vdots & \ddots & \vdots \\
0 & 0 & \cdots & a_{n n}
\end{array}\right) \in \Psi\left(M_{n}(R)\right)
$$

Therefore $\left(a_{i j}\right) \in \Psi\left(M_{n}(R)\right)$, and so $M_{n}(\Psi(R)) \subseteq \Psi\left(M_{n}(R)\right)$, as required.

Proposition 2.4. Let $x, y \in R$. Then $x+y+x y \in u r(R)$ if and only if $x+y+y x \in u r(R)$.

Proof. Suppose that $x+y+x y \in u r(R)$. Then $(x+1)(y+1)-1=x+y+$ $x y \in u r(R)$, and then $1+(x+1)(-y-1) \in u r(R)$. In view of Lemma 2.1, $1+(-y-1)(x+1) \in u r(R)$, whence $(y+1)(x+1)-1 \in u r(R)$. That is, $x+y+y x \in \operatorname{ur}(R)$. The converse is analogous.

Let $e \in R$ be an idempotent and $a \in R$. In [7, Theorem 4], Lam and Murray proved that eae $\in \operatorname{ur}(e R e)$ if and only if eae $+1-e \in u r(R)$. We extend this result as follows.

Proposition 2.5. Let $e \in R$ be an idempotent of a ring $R$ and $a \in R$. Then the following are equivalent:
(1) eae $\in \operatorname{ur}(e R e)$.
(2) $a e+1-e \in u r(R)$.
(3) $e a+1-e \in u r(R)$.

Proof. (1) $\Rightarrow(2)$ Since eae $\in$ ur $(e R e)$, we can find some $u \in U(e R e)$ such that $e a e=($ eae $)($ eue $)(e a e)$. Hence $e a e+1-e=(e a e+1-e)(e u e+1-e)(e a e+1-e)$. Clearly, eue $+1-e \in U(R)$. This means that $1+e(a e-e) \in u r(R)$. It follows from Lemma 2.1 that $1+(a e-e) e \in u r(R)$. That is, ae $+1-e \in u r(R)$.

$(2) \Rightarrow(1)$ As $a e+1-e=1+(a e-e) e \in u r(R)$, by Lemma 2.1, we have $e a e+1-e=1+e(a e-e) \in u r(R)$. It follows from [7, Theorem 4] that eae $\in \operatorname{ur}(e R e)$.

$(1) \Leftrightarrow(3)$ is proved in the same manner. 


\section{Stable range one}

Lemma 3.1. Suppose that $a x+b=1$ in $R$. If $a \in u r(R)$, then there exists $z \in R$ such that $x+z b \in U(R)$.

Proof. Since $a \in \operatorname{ur}(R)$, there exist $e=e^{2} \in R$ and $u \in U(R)$ such that $a=e u$. Hence eux $(1-e)+b(1-e)=1-e$, and then

$$
a+b(1-e) u=(1-\operatorname{eux}(1-e)) u=(1+\operatorname{eux}(1-e))^{-1} u \in U(R) .
$$

By [4, Lemma 3.1], we can find $z \in R$ such that $x+z b \in U(R)$.

Theorem 3.2. $\Psi(R)$ has stable range one.

Proof. Given $a x+b=1$ with $a \in 1+\Psi(R)$ and $x, b \in R$, we can find $c_{1}, \ldots, c_{m} \in$ $R$ such that $a=1+c_{1}+\cdots+c_{m}$ and each $c_{i} \in \Phi(R)$. Hence $\left(1+c_{1}\right) x+\left(c_{2}+\right.$ $\left.\cdots+c_{m}\right) x+b=1$. As $1+c_{1} \in \operatorname{ur}(R)$, by Lemma 3.1, we can find some $z_{1} \in R$ such that

$$
x+z_{1}\left(c_{2}+\cdots+c_{m}\right) x+z_{1} b=u_{1} \in U(R) .
$$

Hence

$$
\left(1+z_{1} c_{2}\right) x u_{1}^{-1}+z_{1}\left(c_{3}+\cdots+c_{m}\right) x u_{1}^{-1}+z_{1} b u_{1}^{-1}=1 .
$$

As $1+z_{1} c_{2} \in u r(R)$, by Lemma 3.1 again, we have $z_{2} \in R$ such that

$$
x u_{1}^{-1}+z_{2} z_{1}\left(c_{3}+\cdots+c_{m}\right) x u_{1}^{-1}+z_{2} z_{1} b u_{1}^{-1} \in U(R),
$$

and then $x+z_{2} z_{1}\left(c_{3}+\cdots+c_{m}\right) x+z_{2} z_{1} b \in U(R)$. By iteration of this process, we have $z \in R$ such that $x+z b \in U(R)$. Therefore $\Psi(R)$ has stable range one.

A right $R$-module $A$ is said to have the finite exchange property if for every right $R$-module $M$ and any two decompositions $M=A^{\prime} \oplus N=\bigoplus_{i \in I} A_{i}$, where $A_{R}^{\prime} \cong A_{R}$ and the index set $I$ is finite, then there exist submodules $A_{i}^{\prime} \subseteq A_{i}$ such that $M=A^{\prime} \oplus\left(\bigoplus_{i \in I} A_{i}^{\prime}\right)$. A ring $R$ is said to be an exchange ring provided that $R$ has the finite exchange property as a right $R$-module. As is well known, a ring $R$ is an exchange ring if and only if for any $x \in R$, there exists an idempotent $e \in R$ such that $e \in x R$ and $1-e \in(1-x) R$ (cf. [8, Proposition 28.6]).

Corollary 3.3. Let $R$ be an exchange ring, and let $A \in M_{n}(\Psi(R))$ be regular. Then A admits a diagonal reduction.

Proof. By virtue of Theorem 3.2, $\Psi(R)$ has stable range one, and then so has $M_{n}(\Psi(R))$ from [7, Corollary 5.4]. Since $A \in M_{n}(\Psi(R))$ is regular, there exists $E=E^{2} \in M_{n}(R)$ such that $A(n R)=E(n R)$. Now we have a split exact sequence

$$
0 \rightarrow \operatorname{Ker} E \rightarrow n R \stackrel{E}{\longrightarrow} E(n R) \rightarrow 0
$$

of right $R$-modules. Thus, $E(n R) \oplus \operatorname{Ker} E \cong n R$, and so $E(n R)$ is a finitely generated projective right $R$-module. As $A \in M_{n}(\Psi(R))$, we see that $E \in$ 
$M_{n}(\Psi(R))$; hence, $E(n R)=E(n R) \Psi(R)$. As in the proof of [8, Exercise 29.9], we can find idempotents $e_{1}, \ldots, e_{n} \in \Psi(R)$ such that

$$
E(n R) \cong e_{1} R \oplus \cdots \oplus e_{n} R \cong \operatorname{diag}\left(e_{1}, \ldots, e_{n}\right)(n R)
$$

as right $R$-modules. This implies that

$$
E\left({ }^{n} R\right) \bigotimes_{R} R^{n} \cong \operatorname{diag}\left(e_{1}, \ldots, e_{n}\right)\left({ }^{n} R\right) \bigotimes_{R} R^{n}
$$

where ${ }^{n} R=\left\{\left(\begin{array}{c}r_{1} \\ \vdots \\ r_{n}\end{array}\right) \mid r_{1}, \ldots, r_{n} \in R\right\}$ and $R^{n}=\left\{\left(r_{1}, \ldots, r_{n}\right) \mid r_{1}, \ldots, r_{n} \in\right.$ $R$ \}. Obviously, ${ }^{n} R \bigotimes_{R} R^{n} \cong M_{n}(R)$. Thus, we infer that $\varphi: A M_{n}(R) \cong$ $\operatorname{diag}\left(e_{1}, \ldots, e_{n}\right) M_{n}(R)$. One easily checks that

$$
M_{n}(R) A=M_{n}(R) \varphi(A) \text { and } \varphi(A) M_{n}(R)=\operatorname{diag}\left(e_{1}, \ldots, e_{n}\right) M_{n}(R) .
$$

Write $A=X \varphi(A)$ and $\varphi(A)=Y A$. Without loss of the generality, we may assume that $X, Y \in M_{n}(\Psi(R))$. Since

$\left(X+\left(I_{n}-X Y\right)\right) Y+\left(I_{n}-X Y\right)\left(I_{n}-Y\right)=I_{n}$ and $X+\left(I_{n}-X Y\right) \in M_{n}(\Psi(R))$ we have $Z \in M_{n}(R)$ such that

$$
\begin{aligned}
U: & =X+\left(I_{n}-X Y\right)\left(I_{n}+\left(I_{n}-Y\right) Z\right) \\
& =X+\left(I_{n}-X Y\right)+\left(I_{n}-X Y\right)\left(I_{n}-Y\right) Z \in \mathrm{GL}_{n}(R) .
\end{aligned}
$$

Thus, it follows by [4, Lemma 3.1] that there exists $W \in M_{n}(R)$ such that $V:=$ $Y+W\left(I_{n}-X Y\right) \in \mathrm{GL}_{n}(R)$. This implies that $V A=Y A=\varphi(A)$. Likewise, we have $V^{\prime} \in \operatorname{GL}_{n}(R)$ such that $\varphi(A) V^{\prime}=\operatorname{diag}\left(e_{1}, \ldots, e_{n}\right)$. Therefore $V A V^{\prime}=$ $\operatorname{diag}\left(e_{1}, \ldots, e_{n}\right)$, as desired.

Corollary 3.4. Let $R$ be an exchange ring, and let $\left(a_{i j}\right) \in M_{n}(R)$ be regular. If each $1+a_{i j} r \in R$ is unit-regular for all $r \in R$, then $\left(a_{i j}\right)$ admits a diagonal reduction.

Proof. In view of Lemma 2.1, $\Phi(R)=\{x \in R \mid 1+x t \in R$ is unit-regular for all $t \in R\}$. If each $1+a_{i j} r \in R$ is unit-regular for all $r \in R$, then $a_{i j} \in \Phi(R)$. Thus, $\left(a_{i j}\right) \in M_{n}(\Psi(R))$. According to Corollary 3.3, we complete the proof.

As is well known, a regular ring $R$ has stable range one if and only if every element in $R$ is unit-regular. The following result gives an analogue for ideals of a regular ring having stable range one.

Theorem 3.5. An ideal $I$ of a regular ring $R$ has stable range one if and only if for any $x \in I, r \in R, 1+x r$ is unit-regular.

Proof. Let $I$ be an ideal of a regular ring $R$. Suppose that $I$ has stable range one. For any $x \in I, r \in R, 1+x r \in 1+I$. Write $1+x r=(1+x r) y(1+x r)$ for 
$y \in R$. It follows from $(1+x r) y+(1-(1+x r) y)=1$ that there exists $z \in R$ such that

$$
u:=1+x r+(1-(1+x r) y) z \in U(R) .
$$

Hence, $1+x r=(1+x r) y u$. Therefore $1+x r=(1+x r) u^{-1}(1+x r)$, and so $1+x r \in R$ is unit-regular.

Conversely, assume that $1+x r$ is unit-regular for all $x \in I, r \in R$. Obviously, $\Phi(R)=\{x \in R \mid 1+x r \in \operatorname{ur}(R)$ for all $r \in R\}$. Let $x, y \in \Phi(R), s, t \in R$. Then $s x t \in \Phi(R)$. Write $1+(x+y) t=(1+(x+y) t) c(1+(x+y) t)$. Then

$$
(1+(x+y) t) c+(1-(1+(x+y) t) c)=1 .
$$

Clearly, $x+y \in \Psi(R)$. According to Theorem 3.2, $\Psi(R)$ has stable range one. As in the proof of $[6$, Theorem 1.8], we see that $v:=c+z(1-(1+(x+y) t) c) \in$ $U(R)$ for $z \in R$. Therefore

$$
1+(x+y) t=(1+(x+y) t) v(1+(x+y) t) .
$$

That is, $1+(x+y) t \in u r(R)$. Thus $x+y \in \Phi(R)$, and so $\Phi(R)$ is an ideal of $R$. We infer that $\Phi(R)=\Psi(R)$. Hence, $\Phi(R)$ has stable range one by Theorem 3.2. In fact, one easily checks that

$$
\begin{aligned}
\Phi(R) & =\sum_{I \unlhd R}\{I \mid I \text { has stable range one }\} \\
& =\{x \in R \mid R x R \text { has stable range one }\} .
\end{aligned}
$$

By hypothesis, $I \subseteq \Phi(R)$, and therefore $I$ has stable range one, as asserted.

Analogously, we deduce that an ideal $I$ of a regular ring $R$ has stable range one if and only if for any $x \in I, r \in R, 1+r x$ is unit-regular. Let $R$ be a regular ring, and let $\left(a_{i j}\right) \in M_{n}(R)$. If $1+a_{i j} r \in R$ is unit-regular for all $r \in R$, then $\left(a_{i j}\right)$ is the product (sum) of an idempotent matrix and an invertible matrix over $R$. Set $I=\sum_{1 \leq i, j \leq n} R a_{i j} R$. Then each $a_{i j} \in \Phi(R)$, and so $R a_{i j} R \subseteq \Psi(R)$. This implies that $I$ has stable range one by Theorem 3.5, and we are done.

\section{4. $\Psi$-regularity}

We say that $a \in R$ is $\Psi$-regular in case there exists some $u \in \Psi(R)$ such that $a=a u a$. Let $I$ be an ideal of a ring $R$. We say that $I$ is $\Psi$-regular in case every element in $I$ is $\Psi$-regular. A ring $R$ is $\Psi$-regular provided that it is $\Psi$-regular as an ideal of itself.

Lemma 4.1. Let $a \in R$. Then $a \in R$ is $\Psi$-regular if and only if there exists some $x \in \Psi(R)$ such that a-axa is $\Psi$-regular.

Proof. Suppose that $a-a x a$ is $\Psi$-regular and $x \in \Psi(R)$. Then we have $u \in$ $\Psi(R)$ such that $a-a x a=(a-a x a) u(a-a x a)$. Hence $a=a(x+(1-x a) u(1-$ $a x)) a$. As $x, u \in \Psi(R)$, we deduce that $x+(1-x a) u(1-a x) \in \Psi(R)$. So $a \in R$ is $\Psi$-regular. The converse is obvious. 
Lemma 4.2. Let $I$ be an ideal of a ring $R$, and let $e \in R$ be an idempotent. If I is $\Psi$-regular, then so is eIe.

Proof. Given any $a \in I$, we have eae $\in I$. Let $I$ be $\Psi$-regular. Then we can find some $x \in \Psi(R)$ such that eae $=($ eae $) x(e a e)$. In addition, we have $x_{1}, \ldots, x_{m} \in$ $\Phi(R)$ such that $x=x_{1}+\cdots+x_{m}$. For any $s, t \in R$, we have $1+(r e)\left(e x_{i} e\right)(e s e) \in$ $u r(R)$. In view of Proposition 2.5, we get $e+($ ere $)\left(e x_{i} e\right)(e s e) \in u r(e R e)$. This implies that each $e x_{i} e \in \Psi(e R e)$, and so exe $=e x_{1} e+\cdots+e x_{m} e \in \Psi(e R e)$. As $e a e=(e a e)(e x e)(e a e)$, we prove that eae $\in e I e$ is $\Psi$-regular, as required.

Theorem 4.3. Let $I$ be an ideal of a ring $R$. If $I$ is $\Psi$-regular, then so is $M_{n}(I)$.

Proof. Given any $\left(\begin{array}{ll}a & b \\ c & d\end{array}\right) \in M_{2}(I)$, we have $b^{\prime} \in \Psi(R)$ such that $b=b b^{\prime} b$. Write $b^{\prime}=h_{1}+\cdots+h_{m}(m \in \mathbb{N})$, where each $h_{i} \in \Phi(R)$. Then $\left(\begin{array}{cc}0 & 0 \\ b^{\prime} & 0\end{array}\right)=\sum_{i=1}^{m}\left(\begin{array}{cc}0 & 0 \\ h_{i} & 0\end{array}\right)$. For any $\left(\begin{array}{ll}r_{11} & r_{12} \\ r_{21} & r_{22}\end{array}\right) \in M_{2}(R)$, we have

$$
I_{2}+\left(\begin{array}{cc}
0 & 0 \\
h_{i} & 0
\end{array}\right)\left(\begin{array}{ll}
r_{11} & r_{12} \\
r_{21} & r_{22}
\end{array}\right)=\left(\begin{array}{cc}
1 & 0 \\
h_{i} r_{11} & 1+h_{i} r_{12}
\end{array}\right) .
$$

Clearly, we can find $U \in \mathrm{GL}_{2}(R)$ such that $\left(\begin{array}{cc}1 & 0 \\ h_{i} r_{11} & 1+h_{i} r_{12}\end{array}\right)=U\left(\begin{array}{ll}1 & 0 \\ 0 & 1+h_{i} r_{12}\end{array}\right)$. So we see that $I_{2}+\left(\begin{array}{cc}0 & 0 \\ h_{i} & 0\end{array}\right)\left(\begin{array}{cc}r_{11} & r_{12} \\ r_{21} & r_{22}\end{array}\right) \in u r\left(M_{2}(R)\right)$. As a result, $\left(\begin{array}{cc}0 & 0 \\ b^{\prime} & 0\end{array}\right) \in \Psi\left(M_{2}(R)\right)$. By virtue of Lemma 4.1, it suffices to prove that

$$
\left(\begin{array}{ll}
a & b \\
c & d
\end{array}\right)-\left(\begin{array}{ll}
a & b \\
c & d
\end{array}\right)\left(\begin{array}{cc}
0 & 0 \\
b^{\prime} & 0
\end{array}\right)\left(\begin{array}{ll}
a & b \\
c & d
\end{array}\right) \in M_{2}(I)
$$

is $\Psi$-regular. That is, it suffices to prove that $\left(\begin{array}{cc}a^{\prime} & 0 \\ c^{\prime} & d^{\prime}\end{array}\right) \in M_{2}(I)$ is $\Psi$-regular. Clearly, we have $a^{\prime \prime}, d^{\prime \prime} \in \Psi(R)$ such that $a^{\prime}=a^{\prime} a^{\prime \prime} a^{\prime}$ and $d^{\prime}=d^{\prime} d^{\prime \prime} d^{\prime}$. According Theorem 2.3, we see that $\left(\begin{array}{cc}a^{\prime \prime} & 0 \\ 0 & d^{\prime \prime}\end{array}\right) \in \Psi\left(M_{2}(I)\right)$, and so it suffices to prove that

$$
\left(\begin{array}{cc}
a^{\prime} & 0 \\
c^{\prime} & d^{\prime}
\end{array}\right)-\left(\begin{array}{cc}
a^{\prime} & 0 \\
c^{\prime} & d^{\prime}
\end{array}\right)\left(\begin{array}{cc}
a^{\prime \prime} & 0 \\
0 & d^{\prime \prime}
\end{array}\right)\left(\begin{array}{cc}
a^{\prime} & 0 \\
c^{\prime} & d^{\prime}
\end{array}\right)=\left(\begin{array}{cc}
0 & 0 \\
c^{\prime \prime} & 0
\end{array}\right) \in M_{2}(I)
$$

is $\Psi$-regular. Obviously, we have $u \in \Psi(R)$ such that $c^{\prime \prime}=c^{\prime \prime} u c^{\prime \prime}$. Hence

$$
\left(\begin{array}{cc}
0 & 0 \\
c^{\prime \prime} & 0
\end{array}\right)=\left(\begin{array}{cc}
0 & 0 \\
c^{\prime \prime} & 0
\end{array}\right)\left(\begin{array}{ll}
0 & u \\
0 & 0
\end{array}\right)\left(\begin{array}{cc}
0 & 0 \\
c^{\prime \prime} & 0
\end{array}\right) \text {. }
$$

As $u \in \Psi(R)$, it follows from Theorem 2.3 that $\left(\begin{array}{ll}0 & u \\ 0 & 0\end{array}\right) \in \Psi\left(M_{2}(R)\right)$. We infer that $\left(\begin{array}{cc}0 & 0 \\ c^{\prime \prime} & 0\end{array}\right)$ is $\Psi$-regular. Consequently, $M_{2}(I)$ is $\Psi$-regular. By induction, $M_{2^{n}}(I)$ is $\Psi$-regular. Choose $E=\operatorname{diag}\left(I_{n}, 0, \ldots, 0\right)_{2^{n} \times 2^{n}}$. Therefore $M_{n}(I) \cong$ $E M_{2^{n}}(I) E$ is $\Psi$-regular from Lemma 4.2.

Proposition 4.4. A ring $R$ is $\Psi$-regular if and only it is unit-regular.

Proof. Suppose that $R$ is unit-regular. Given any $a \in R$, there exists $x \in R$ such that $a=a x a$. Clearly, $1+r x s \in u r(R)$ for all $r, s \in R$. Hence $x \in \Psi(R)$. This infers that $R$ is $\Psi$-regular. 
Conversely, assume that $R$ is $\Psi$-regular. Given any $a \in R$, there exists $x \in \Psi(R)$ such that $a=$ axa. Obviously, $(x+(1-x a)) a+(1-x a)(1-a)=1$. Since $x+(1-x a) \in 1+\Psi(R)$, by Theorem 3.2, there exists $y \in R$ such that $x+(1-x a)+(1-x a)(1-a) y \in U(R)$. That is, $u:=x+(1-x a)(1+(1-a) y) \in$ $U(R)$. Therefore $a=a x a=a u a$, as desired.

Corollary 4.5. If $R$ is unit-regular, then so is $M_{n}(R)$ for all $n \in \mathbb{N}$.

Proof. According to Proposition 4.4 and Theorem 4.3, we complete the proof.

We note that Corollary 4.5 is a well-known result in the theory of unit-regular rings. However, the only known proof of it depends on cancelation of modules (cf. [5, Theorem 4.5]). Our treatment above provided the first element-wise proof of this result.

Acknowledgements. The author would like to thank the referee for his/her corrections, which lead to the new version of this paper.

\section{References}

[1] P. Ara, K. R. Goodearl, K. C. O'Meara, and E. Pardo, Diagonalization of matrices over regular rings, Linear Algebra Appl. 265 (1997), 147-163.

[2] _ Separative cancellation for projective modules over exchange rings, Israel J. Math. 105 (1998), 105-137.

[3] B. Brown and N. H. Mccoy, The maximal regular ideal of a ring, Proc. Amer. Math. Soc. 1 (1950), 165-171.

[4] K. R. Goodearl, Cancellation of low-rank vector bundles, Pacific J. Math. 113 (1984), no. 2, 289-302.

[5] _ von Neumann Regular Rings, Second edition. Robert E. Krieger Publishing Co., Inc., Malabar, FL, 1991.

[6] T. Y. Lam, A crash course on stable range, cancellation, substitution and exchange, J. Algebra Appl. 3 (2004), no. 3, 301-343.

[7] T. Y. Lam and W. Murray, Unit regular elements in corner rings, Bull. Hong Kong Math. Soc. 1 (1997), no. 1, 61-65.

[8] A. A. Tuganbaev, Rings Close to Regular, Mathematics and its Applications, 545. Kluwer Academic Publishers, Dordrecht, 2002.

Department of Mathematics

HANGZHOU NORMAL UNIVERSITY

Hangzhou 310036, P. R. China

E-mail address: huanyinchen@yahoo.cn 